\title{
Perception of radiation dose and potential risks of computed tomography in emergency department medical personnel
}

\author{
Jin Hee Lee', Kyuseok Kim¹, Kyoung Ho Lee², Kwang Pyo Kim³, \\ Yu Jin Kim ${ }^{1}$, Chanjong Park ${ }^{4}$, Changwoo Kang ${ }^{5}$, Soo Hoon Lee ${ }^{5}$, \\ Jin Hee Jeong ${ }^{5}$, Joong Eui Rhee ${ }^{6}$
}

\author{
'Department of Emergency Medicine, Seoul National University Hospital, Seoul National University College \\ of Medicine, Seoul, Korea \\ ${ }^{2}$ Department of Radiology, Seoul National University Bundang Hospital, Seoul National University College \\ of Medicine, Seongnam, Korea \\ ${ }^{3}$ Department of Nuclear Engineering, Kyung Hee University, Yongin, Korea \\ ${ }^{4}$ Department of Emergency Medicine, Veterans Health Service Medical Center, Seoul, Korea \\ ${ }^{5}$ Department of Emergency Medicine, Gyeongsang National University Hospital, Jinju, Korea \\ ${ }^{6}$ Department of Emergency Medicine, Samsung Medical Center, Seoul, Korea
}

Objective Use of computed tomography (CT) continues to increase, but the relatively high radiation doses associated with CT have raised health concerns such as future risk of cancer. We investigated the level of awareness regarding radiation doses and possible risks associated with CT in medical personnel (MP).

Methods This study was conducted from April to May 2012 and included physicians and nurses who worked in the emergency department of 17 training hospitals. The questionnaire included measurement of the effect of CT or radiography on health using a 10-point numerical rating scale, estimation of the radiation dose of one abdominal CT scan compared with one chest radiograph, and perception of the increased lifetime risk of cancer associated with CT.

Results A total of 354 MP participated in this study: 142 nurses, 87 interns, 86 residents, and 39 specialists. Interns were less aware of the effects of CT or radiography on health than other physicians or nurses (mean \pm SD of $4.8 \pm 2.7,5.9 \pm 2.7,6.1 \pm 2.7$, and $6.0 \pm 2.2$ for interns, residents, specialists, and nurses, respectively; $\mathrm{P}<0.05)$. There was a significant difference in knowledge about the relative radiation dose of one abdominal CT scan compared with one chest radiograph between physicians and nurses (48.6\% vs. $28.9 \%$ for physicians vs. nurses, $\mathrm{P}<0.05$ ). MP perceived an increased risk of cancer from radiation associated with CT.

Conclusion MP perceive the risk of radiation associated with $\mathrm{CT}$, but their level of knowledge seems to be insufficient.

Keywords Radiation injuries; Computed tomography; Perception
elSSN: 2383-4625

Received: 11 July 2014

Revised: 22 August 2014

Accepted: 17 September 2014

Correspondence to: Kyuseok Kim Department of Emergency Medicine, Seoul National University Bundang Hospital, 82 Gumi-ro 173beon-gil, Bundang-gu, Seongnam 463-707, Korea

E-mail: dremkks@snubh.org

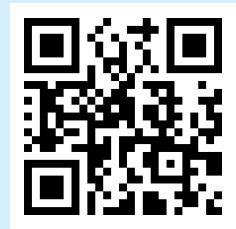

How to cite this article: Lee JH, Kim K, Lee KH, Kim KP, Kim YJ, Park C, Kang C, Lee SH, Jeong JH, Rhee JE. Perception of radiation dose and potential risks of computed tomography in emergency department medical personnel. Clin Exp Emerg Med 2015;2(2):123-129.

This is an Open Access article distributed under the terms of the Creative Commons Attribution Non-Commercial License (http:// creativecommons.org/licenses/by-nc/3.0/). 


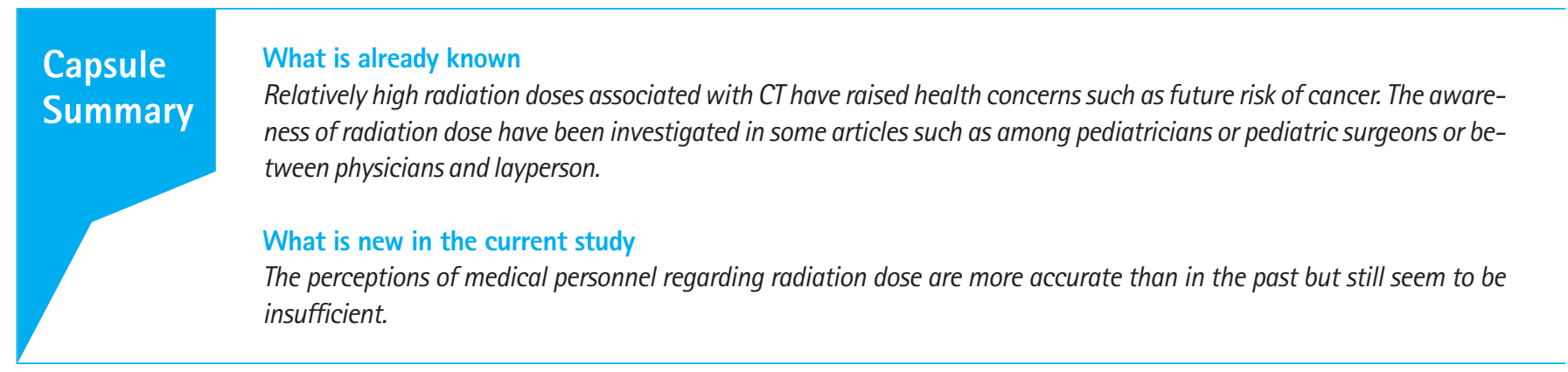

\section{INTRODUCTION}

The use of computed tomography (CT) has increased rapidly up until the early 2000s, in part as a result of advances in CT technology. ${ }^{1}$ CT imaging is a valuable diagnostic technique, but the relatively high radiation doses associated with $\mathrm{CT}$ have raised health concerns such as future risk of cancer. ${ }^{1,2}$ In the United States, there has been an apparent decline in use of CT since 2008; the investigators in one study explained that this might be attributable to public awareness and published studies regarding the risks of radiation. ${ }^{3}$

In several studies on awareness of radiation doses and possible risks, emergency physicians, radiologists, pediatricians, and pediatric surgeons were unable to provide accurate estimates of radiation doses for $\mathrm{CT}$ and their level of awareness of the hazards of radiation was generally low. ${ }^{4-7}$ However, previous studies targeted only a small number of physicians or patients, so the results may not reflect the opinions of other medical personnel, such as nurses and interns.

In this study, we investigated the level of awareness regarding radiation doses and possible risks associated with $\mathrm{CT}$ among nurses, interns, residents, and specialists in the emergency department (ED).

\section{METHODS}

\section{Participants}

This study was a cross-sectional survey conducted from April to May 2012. A survey was designed and then distributed to medical personnel, including interns, emergency physicians (residents and specialists), and nurses who worked in the ED of 17 training hospitals.

This study was approved by the research ethics board at the Seoul National University Bundang Hospital.

\section{Survey structure}

The questionnaire (Appendix) included demographic characteristics (01 and 02$)$, measurement of the effect of $\mathrm{CT}$ or radiography on health using a 10-point numerical rating scale ("none" on the left side and "the most possible" on the right side) (03), estimation of the radiation dose for one abdominal CT scan compared with one chest radiograph (04), measurement of the belief that the lifetime risk of cancer increases with radiation exposure for medical purposes (05), and perception of the increased lifetime risk of cancer with $\mathrm{CT}$ scan in different cases (06 to Q9). The questions about lifetime risk of cancer associated with use of CT were developed on the basis of a reference article. 'The last 2 questions addressed previous knowledge regarding the risk of radiation exposure due to medical purposes and the route by which this information was obtained (010 and $\mathrm{Q11}$ ).

Responses were elicited through multiple choice questions except $\mathrm{O} 3$ (measurement of the effect of $\mathrm{CT}$ or radiography on health).

\section{Data analysis}

Data were analyzed using the statistical package PASW Statistics ver. 18.0 (SPSS Inc., Chicago, IL, USA). Values for continuous vari-

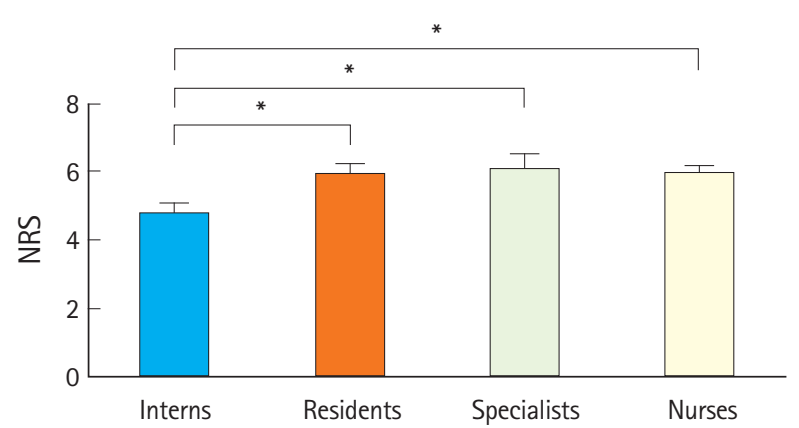

Fig. 1. The possibility of computed tomography or radiography affecting health was rated most highly by specialists $(6.1 \pm 2.7)$, followed by nurses, residents, and interns, respectively $(6.0 \pm 2.2,5.9 \pm 2.7$, and $4.8 \pm$ 2.7). There were significant differences between interns and other medical personnel. NRS, numerical rating scale. ${ }^{*} \mathrm{P}<0.05$. 
ables are expressed as medians (interquartile range), and categorical variables are expressed as numbers (percentages). The MannWhitney U-test and Wilcoxon signed rank test were used to compare differences between the 2 groups. Fisher exact test and Pearson chi-square test were used for categorical variables. Two sided P-value of $<0.05$ was considered statistically significant.

\section{RESULTS}

The questionnaire were completed by $354(34.3 \%)$ of the 1,032 subjects who were invited to participate in the study. In total, 154 $(43.5 \%)$ of the respondents were male, and the mean age was $29.5 \pm 5.2$ years. Most of the respondents were nurses (40.1\%), followed by residents $(24.6 \%)$, interns $(24.3 \%)$, and specialists (11.0\%).

The effect of $\mathrm{CT}$ or radiography on health was rated lowest by

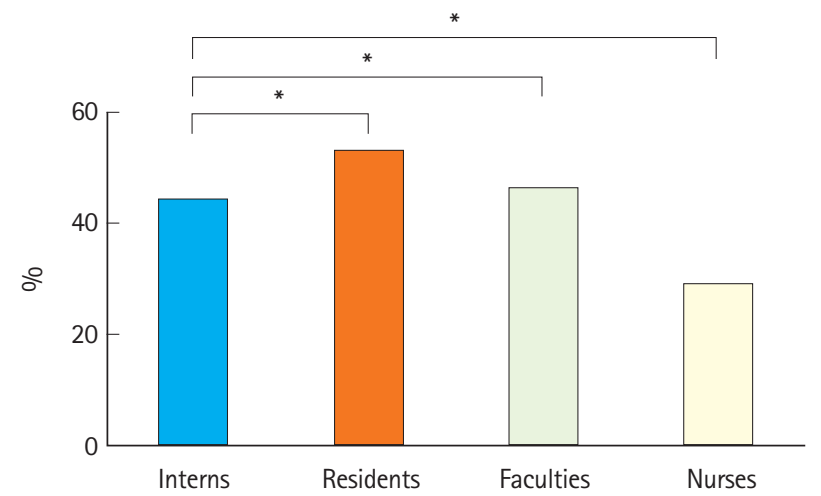

Fig. 2. Dose estimates for one abdominal computed tomography scan versus one chest radiograph. Only 41 nurses (28.9\%), 38 interns (44.2\%), 46 residents (53.5\%), and 18 specialists (47.4\%) provided the correct answer. Knowledge about the relative radiation dose was also significantly different between physicians and nurses (48.6\% vs. $28.9 \%$, respectively). ${ }^{*} \mathrm{P}<0.05$. interns (03) (mean \pm SD of $4.8 \pm 2.7,5.9 \pm 2.7,6.1 \pm 2.7$, and $6.0 \pm 2.2$ for interns, residents, specialists, and nurses, respectively) (Fig. 1). Regarding the relative radiation dose for one abdominal CT scan compared with one chest radiograph, only 143 participants (40.4\%) replied that the radiation dose for CT was equivalent to that for 1,000 chest radiographs, and there was a significant difference in knowledge about the relative radiation dose for one abdominal CT scan compared with one chest radiograph between physicians and nurses (44.2\%, 53.5\%, 47.4\%, and 28.9\% for interns, residents, specialists, and nurses, respectively, $\mathrm{P}<0.05$ ) (Fig. 2). In addition, $49.2 \%$ of respondents underestimated the radiation dose for CT (Table 1).

Most respondents (81.0\%) believed that the lifetime risk of cancer was increased as a result of exposure to radiation for medical purposes (Table 1). And most respondents (84.3\%) answered that they already knew the risk of radiation exposure for medical purposes; specialists were most knowledgeable about this risk (Table 1). The participants generally gained this knowledge in the hospital (37.2\%), followed by mass media (20.6\%).

The proportions of correct answers in the different cases (06 to 09 ) were $25.4 \%, 35.4 \%, 24.3 \%$, and $15.1 \%$ for interns, residents, specialists, and nurses, respectively, and there was no significant difference among groups (Fig. 3). The proportions of underestimation of the lifetime risk of cancer with use of $\mathrm{CT}$ in the different cases were $64.0 \%, 42.9 \%, 66.6 \%$, and $76.9 \%$, respectively (Fig. 4). Physicians demonstrated greater underestimation of the lifetime risk of cancer in 40-year-old patients (second and fourth cases) as compared with nurses $(P<0.05)$.

\section{DISCUSSION}

The use of CT has increased rapidly, in part because of the advent of fast helical CT, but the relatively higher radiation doses of CT

Table 1. Radiation dose and question about increased risk of cancer

\begin{tabular}{|c|c|c|c|c|c|c|}
\hline & Nurses (142) & Interns (86) & Residents (87) & Specialists (39) & Total (354) & P-value \\
\hline \multicolumn{6}{|c|}{ Dose estimates for one abdominal CT scan vs. one $\mathrm{CR}$} & $<0.01$ \\
\hline $\mathrm{CT}=\mathrm{CR}$ & $0(0)$ & $1(1.2)$ & $0(0)$ & $0(0)$ & $1(0.3)$ & \\
\hline $\mathrm{CT}=10 \mathrm{CR}$ & $29(20.4)$ & $6(7.0)$ & $3(3.5)$ & $2(5.3)$ & $40(11.4)$ & \\
\hline $\mathrm{CT}=100 \mathrm{CR}$ & $54(38.0)$ & $30(34.9)$ & $34(39.5)$ & $14(36.8)$ & $132(37.5)$ & \\
\hline $\mathrm{CT}=1,000 \mathrm{CR}$ & $41(28.9)$ & $38(44.2)$ & $46(53.5)$ & $18(47.4)$ & $143(40.6)$ & \\
\hline $\mathrm{CT}=10,000 \mathrm{CR}$ & 18 (12.7) & $11(12.8)$ & $3(3.5)$ & $4(10.5)$ & $36(10.2)$ & \\
\hline \multicolumn{6}{|c|}{ Lifetime risk of cancer believed to be increased by radiation exposure due to medical purpose } & 0.12 \\
\hline Yes & $115(81.6)$ & $61(70.9)$ & $76(88.4)$ & $33(84.6)$ & $285(81.0)$ & \\
\hline No & $15(10.6)$ & $16(18.6)$ & $8(9.3)$ & $4(10.3)$ & $43(12.2)$ & \\
\hline Don't know & $11(7.8)$ & $9(10.5)$ & $2(2.3)$ & $2(5.1)$ & $24(6.8)$ & \\
\hline Already known radiation risk & $111(78.7)$ & $69(82.1)$ & $78(90.7)$ & $37(94.9)$ & $295(84.3)$ & 0.02 \\
\hline
\end{tabular}

$\mathrm{CT}$, computed tomography; $\mathrm{CR}$, chest radiograph. 

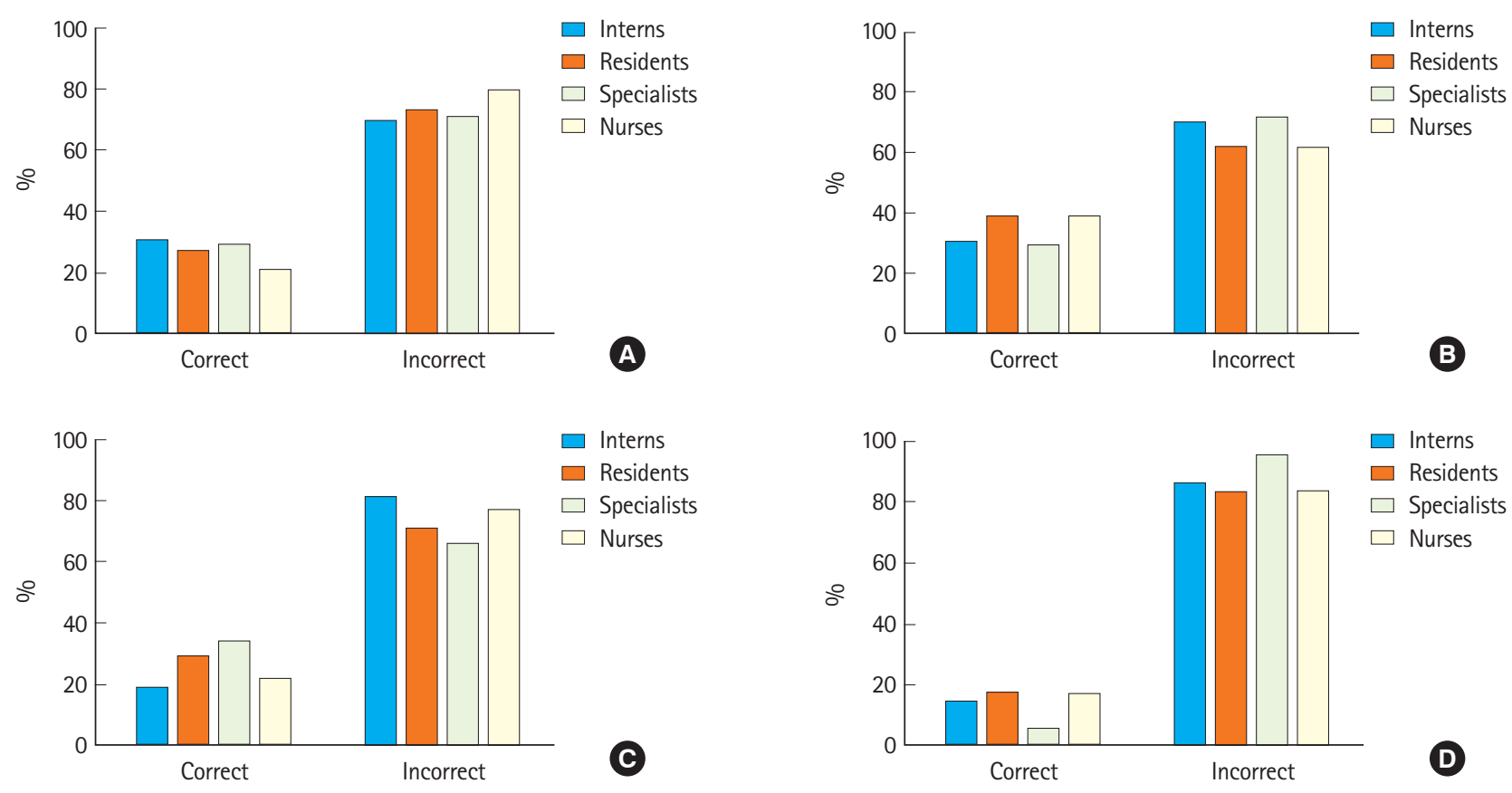

Fig. 3. Perceptions of the increased lifetime risk of cancer with computed tomography (CT) in specific cases (06 to 09). The first case (A) was the current estimate of the excess lifetime risk of brain cancer for a 1-year-old patient undergoing CT scan of the brain, the second case (B) was the current estimate of the excess lifetime risk of brain cancer for a 40-year-old patient undergoing CT scan of the brain, the third case (C) was the current estimate of the excess lifetime risk of cancer for a 1-year-old patient undergoing abdominal CT scan, and the fourth case (D) was the current estimate of the excess lifetime risk of cancer for a 40-year-old patient undergoing abdominal CT scan. A small number of medical personnel answered correctly. The percentage of correct answers for the first, second, third and fourth cases was not significantly different between groups $(P=0.39, P=0.45, P=0.18$, and $P=0.30$, respectively).

result in an increased lifetime risk of cancer mortality attributable to radiation exposure, especially in children. ${ }^{8}$ The possibility of an increased risk of cancer associated with diagnostic CT has been debatable because no epidemiological studies have convincingly shown an increased incidence of cancer associated with low-dose radiation from medical imaging during childhood and adulthood. However, a recently published cohort study showed an increased incidence in leukemia and brain tumor in children and young adults who received more than certain doses of radiation during $\mathrm{CT}_{1}{ }^{2}$ and more research is currently under way. ${ }^{9}$ With this background, efforts to reduce radiation doses should be extensive, and education for medical personnel such as nurses and physicians might be the first step. However, before providing education, there is a need for knowledge about the radiation hazards of $\mathrm{CT}_{\text {, and sever- }}$ al survey studies have been performed to determine how many medical personnels are aware of the exact radiation dose and potential risk associated with $\mathrm{CT}^{4-7}$

Lee et al. ${ }^{4}$ investigated the level of awareness concerning radiation dose and possible risks associated with $\mathrm{CT}$ among emergency physicians, radiologists, and patients. They reported that $45 \%$ of radiologists, 9\% of emergency physicians, and 3\% of patients believe there is an increased risk of cancer from CT. They also iden- tified that patients, more than emergency physicians or radiologists, underestimated the relative radiation dose for one CT scan compared with one chest radiograph. In our study, medical personnel (81.0\%) were aware of the increased risk of cancer associated with $\mathrm{CT}$; however, 49.2\% still underestimated the radiation dose from abdominal CT scan compared with chest radiography.

Thomas et al. ${ }^{5}$ investigated awareness of radiation dose among pediatricians, and 48\% (105/220) of respondents were aware of the article on pediatric CT and radiation. However, only $6 \%$ were correct in their estimate of the quoted lifetime excess cancer risk associated with radiation doses equivalent to pediatric CT. Many $(87 \%)$ of the respondents underestimated the effective doses of various pediatric radiological investigations in chest radiograph equivalents. Although approximately one-half (54\%) of pediatric surgeons believed that the lifetime risk of cancer was increased because of radiation from one abdominal/pelvic CT scan, more than $75 \%$ of respondents underestimated the radiation dose from a CT scan compared with a chest radiograph. ${ }^{6}$ Lee et al. ${ }^{7}$ recently performed research comparing radiologists with non-radiologists about knowledge of radiation exposure in common radiological investigations; they concluded that more radiologists (40\%) had accurate knowledge of radiation doses than non-radiologists (16\%), 

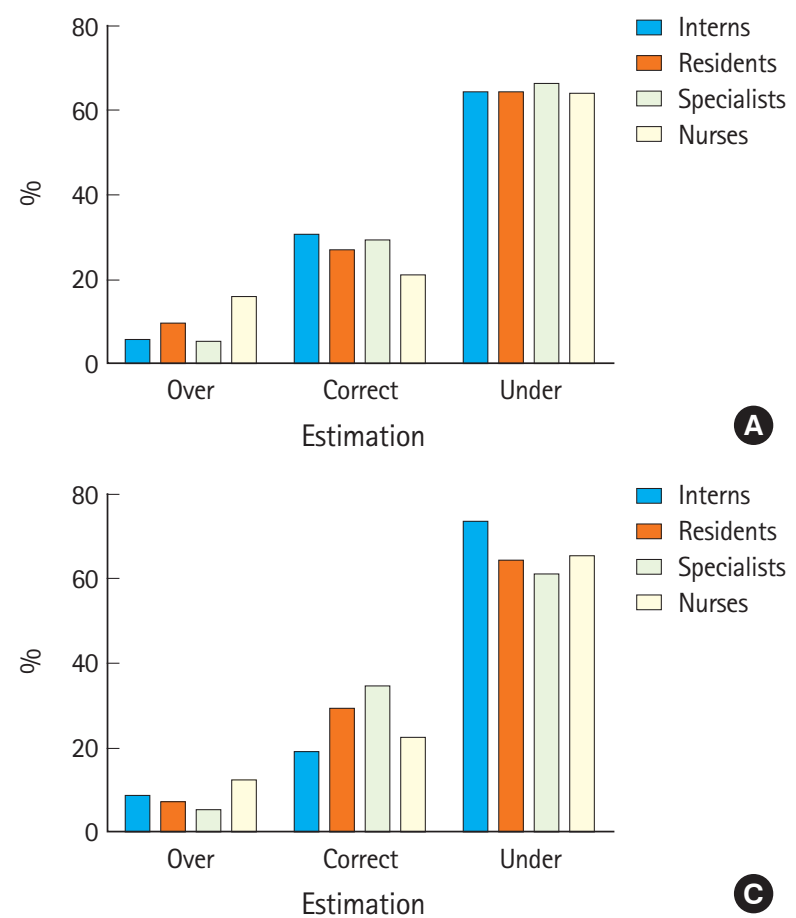
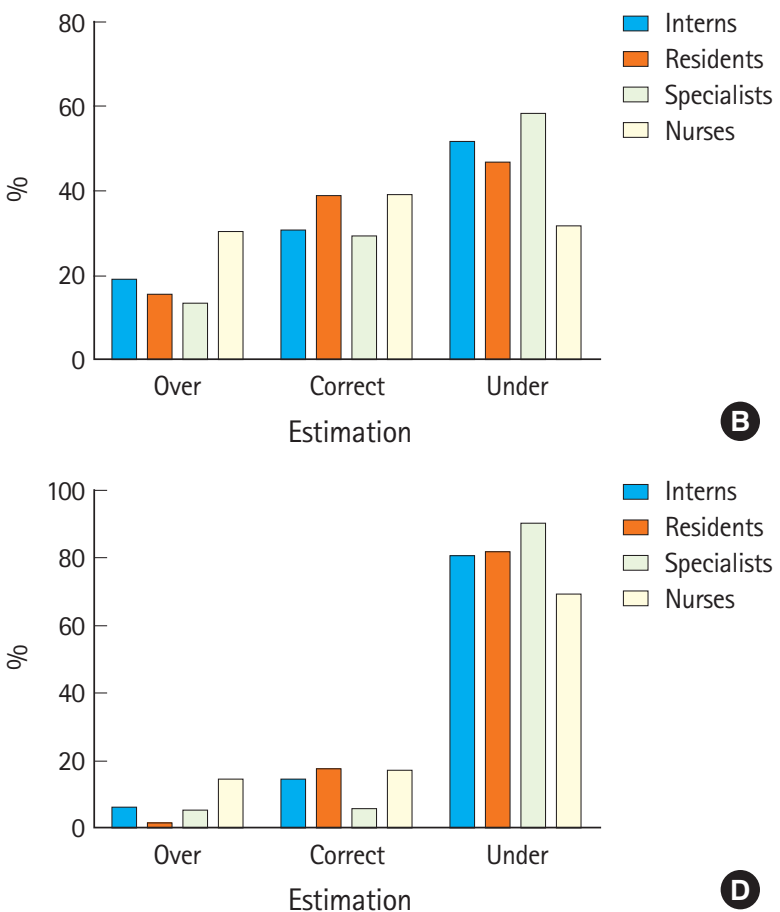

Fig. 4. Perceptions of the increased lifetime risk of cancer with computed tomography (CT) in specific cases (06 to 09). The first case (A) was the current estimate of the excess lifetime risk of brain cancer for a 1-year-old patient undergoing CT scan of the brain, the second case (B) was the current estimate of the excess lifetime risk of brain cancer for a 40-year-old patient undergoing CT scan of the brain, the third case (C) was the current estimate of the excess lifetime risk of cancer for a 1-year-old patient undergoing abdominal CT scan, and the fourth case (D) was the current estimate of the excess lifetime risk of cancer for a 40-year-old patient undergoing abdominal CT scan. The proportions of underestimation of the lifetime risk of cancer for CT in each case were $64.0 \%, 42.9 \%, 66.6 \%$, and $76.9 \%$, respectively. Physicians demonstrated greater underestimation of the lifetime risk of cancer in 40 -year-old patients (second and fourth cases) as compared with nurses $(\mathrm{P}<0.05)$.

but knowledge of radiation doses was generally inadequate in both groups. In our study, $40.6 \%$ of medical personnel correctly estimated the effective dose of one abdominal CT compared with one chest radiograph, but only a few respondents $(25.4 \%, 35.4 \%$, $24.3 \%$, and $15.1 \%$ in each case) correctly estimated the quoted lifetime risk of cancer associated with radiation doses equivalent to another case, and many respondents $(64.0 \%, 42.9 \%, 66.6 \%$, and $76.9 \%$ in each case) underestimated the lifetime risk of cancer associated with CT. However, these results were better than those in previously reported studies. We could infer that these differences originate from more knowledge over time.

Taken together, perceptions about the radiation hazards of CT have increased among medical personnel, but further education is required regarding these hazards.

Supporting increased perceptions about radiation hazards, utilization of CT in pediatric patients in the ED has recently declined in Korea and the United States., ${ }^{3,10}$ However, use of CT in adults in the ED has increased at a rate that far exceeds the growth of ED patient volume. ${ }^{11}$ The authors explained this discrepancy with increasing concerns about radiation exposure in the pediatric population and educational efforts regarding limiting use of $\mathrm{CT}$ in pe- diatric patients. ${ }^{11}$ We have tried to reduce radiation exposure in young patients in various ways, such as educating others by giving lectures at academic conferences in the emergency medicine community.

Several studies have also attempted to reduce the radiation dose with $\mathrm{CT}$ in children and young adults by using another approach. We showed that low-dose CT was non-inferior to standard-dose $\mathrm{CT}$ with respect to negative appendectomy rates in young adults with suspected appendicitis. ${ }^{12}$ Others have shown that CT was avoided in 53\% of participants with use of the staged ultrasonography and CT imaging protocol for the accurate diagnosis of suspected appendicitis in children. ${ }^{13}$

This study has some limitations. First, this survey targeted nurses and physicians in the ED. Emergency medical personnel have recently been educated about the radiation risk of CT in children, so emergency physicians might be more aware of radiation risk than physicians in other departments. Therefore, these findings are not generally applicable. Additional research should be conducted that targets physicians in other departments and the general public. The low response rate also introduces selection bias.

In conclusion, the perceptions of medical personnel regarding 
radiation dose are more accurate than in the past but still seem to be insufficient. Therefore, medical personnel such as nurses and physicians should be better educated about the lifetime risk of cancer associated with $\mathrm{CT}$ to reduce the hazards of radiation from $\mathrm{CT}$ as much as possible.

\section{CONFLICT OF INTEREST}

No potential conflict of interest relevant to this article was reported.

\section{ACKNOWLEDGMENTS}

This study was supported by grant number 11-2009-011 from Seoul National University Bundang Hospital research fund.

\section{REFERENCES}

1. Brenner DJ, Hall EJ. Computed tomography: an increasing source of radiation exposure. N Engl J Med 2007;357:227784.

2. Pearce MS, Salotti JA, Little MP, et al. Radiation exposure from CT scans in childhood and subsequent risk of leukaemia and brain tumours: a retrospective cohort study. Lancet 2012;380: 499-505.

3. Menoch MJ, Hirsh DA, Khan NS, Simon HK, Sturm JJ. Trends in computed tomography utilization in the pediatric emergency department. Pediatrics 2012;129:e690-7.

4. Lee $\mathrm{Cl}$, Haims $\mathrm{AH}$, Monico EP, Brink JA, Forman HP. Diagnostic CT scans: assessment of patient, physician, and radiologist awareness of radiation dose and possible risks. Radiology 2004;
231:393-8.

5. Thomas KE, Parnell-Parmley JE, Haidar S, et al. Assessment of radiation dose awareness among pediatricians. Pediatr Radiol 2006;36:823-32.

6. Rice HE, Frush DP, Harker MJ, Farmer D, Waldhausen JH; APSA Education Committee. Peer assessment of pediatric surgeons for potential risks of radiation exposure from computed tomography scans. J Pediatr Surg 2007;42:1157-64.

7. Lee RK, Chu WC, Graham CA, Rainer TH, Ahuja AT. Knowledge of radiation exposure in common radiological investigations: a comparison between radiologists and non-radiologists. Emerg Med J 2012;29:306-8.

8. Brenner D, Elliston C, Hall E, Berdon W. Estimated risks of radiation-induced fatal cancer from pediatric CT. AJR Am J Roentgenol 2001;176:289-96.

9. Einstein AJ. Beyond the bombs: cancer risks of low-dose medical radiation. Lancet 2012;380:455-7.

10. Oh HY, Kim EY, Kim JE, et al. Trends of CT use in the pediatric emergency department in a tertiary academic hospital of Korea during 2001-2010. Korean J Radiol 2012;13:771-5.

11. Oh HY, Kim EY, Cho J, et al. Trends of CT use in the adult emergency department in a tertiary academic hospital of Korea during 2001-2010. Korean J Radiol 2012;13:536-40.

12. Kim K, Kim YH, Kim SY, et al. Low-dose abdominal CT for evaluating suspected appendicitis. N Engl J Med 2012;366:1596605.

13. Krishnamoorthi $R$, Ramarajan $N$, Wang $N E$, et al. Effectiveness of a staged US and CT protocol for the diagnosis of pediatric appendicitis: reducing radiation exposure in the age of ALARA. Radiology 2011;259:231-9. 
Jin Hee Lee, et al.

Appendix 1. Questionnaire

1. Your gender and age?

Gender: $\quad \square$ male $/ \square$ female Age: $\quad$ ) years

2. What's your specialty?

3. How do you think computed tomography (CT) scans or radiography affect health?

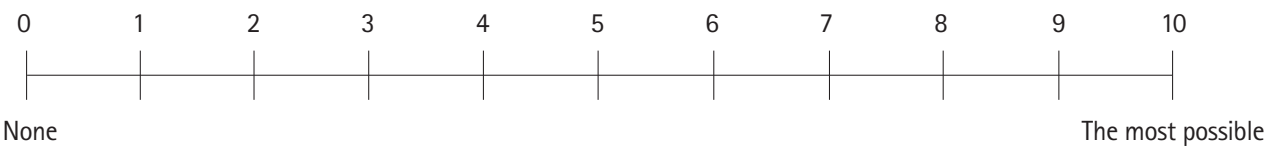

4. Dose estimates for one abdominal CT scan vs. one chest radiograph (CR)?
$\square 1 \mathrm{CR}$
$\square 10 \mathrm{CR}$
$\square 100 \mathrm{CR}$
$\square 1,000 \mathrm{CR}$
$10,000 \mathrm{CR}$

5. Do you believe the lifetime risk of cancer to be increased by radiation exposure due to medical purpose?
$\square$ Yes
$\square$ No
$\square$ Unknown

6. The current estimate of the excess lifetime brain cancer risk of a 1-year-old undergoing brain $\mathrm{CT}$ scan is approximately
$\square 7$ in 100
$\square 7$ in 1,000
$\square 7$ in 10,000
$\square 7$ in 100,000
$\square 7$ in $1,000,000$

7. The current estimate of the excess lifetime brain cancer risk of a 40-year-old undergoing brain CT scan is approximately
$\square 5$ in 100
$\square 5$ in 1,000
$\square 5$ in 10,000
$\square 5$ in 100,000
5 in $1,000,000$

8. The current estimate of the excess lifetime cancer risk of a 1-year-old undergoing abdominal CT scan is approximately
$\square 14$ in 100
$\square 14$ in 1,000
$\square 14$ in 10,000
$\square 14$ in 100,000
14 in $1,000,000$

9. The current estimate of the excess lifetime cancer risk of a 40 -year-old undergoing abdominal CT scan is approximately
$\square 2$ in 100
$\square 2$ in 1,000
$\square 2$ in 10,000
$\square 2$ in 100,000
$\square 2$ in $1,000,000$

10. Have you known previously about the risks of radiation exposure due to medical purpose?
$\square$ Yes
$\square$ No

11. How did you know about it?

$\begin{array}{ll}\square \text { Listened to people around me } & \square \text { Mass media } \\ \square \text { In hospital } & \square \text { Internet }\end{array}$

$\square$ In hospit

Internet 